\title{
Bienestar espiritual en el ámbito hospitalario: aportes para la humanización en salud
}

\author{
Olga Janeth Gómez Ramírez ${ }^{\mathbf{a}}$ - Ángela María Henao Castañob - Alejandra \\ Fuentes Ramírez ${ }^{c}$ - Luz Patricia Díaz Herediad - María Luisa Molano-Pirazáne
}

Resumen: El objetivo del presente artículo es determinar el bienestar espiritual (BE) del personal de enfermería y su aporte ético a la humanización en salud. El método seguido corresponde al enfoque cuantitativo, descriptivo, transversal. El muestreo es no probabilístico, constituido por 148 enfermeros de dos instituciones de salud de la ciudad de Bogotá (A y B). Se aplicó instrumento SHALOM-3 para medir el bienestar espiritual (disonancia y armonía) en los dominios personal, trascendental, ambiental y comunal. En cuanto a los resultados, la evaluación del BE mostró un alto porcentaje de armonía en las dos instituciones; sin embargo, se encontraron porcentajes de disonancia en la institución B, en los dominios trascendental (16\% vs $22 \%$ ), ambiental (12\% vs $19 \%$ ) y personal (11\% vs $18 \%$ ). Se destaca la

a Doctora en enfermería de la Universidad Nacional de Colombia. Profesora asociada de la Facultad de Enfermería de la Universidad Nacional de Colombia. Bogotá, Colombia.

Correo electrónico: ojgomezr@unal.edu.co

ORCID: https://orcid.org/0000-0002-9160-4170

b Doctora en enfermería de la Universidad Nacional de Colombia. Profesora Asistente de la Facultad de Enfermería de la Universidad Nacional de Colombia. Bogotá, Colombia.

Correo electrónico: angmhenaocas@unal.edu.co

ORCID: https://orcid.org/0000-0003-4203-0016

c Doctora en enfermería de la Universidad Nacional de Colombia. Profesora Asistente de la Facultad de Enfermería de la Universidad Nacional de Colombia. Bogotá, Colombia.

Correo electrónico: alejandra.fuentes@unisabana.edu.co

ORCID: https://orcid.org/0000-0003-3311-9211

d Doctora en enfermería de la Universidad Nacional de Colombia. Profesora Asociada de la Universidad Nacional de Colombia. Bogotá, Colombia.

Correo electrónico: Ipdiazh@unal.edu.co

ORCID: https://orcid.org/0000-0002-7167-282X

e Doctora en enfermería de la Universidad Nacional de Colombia. Profesora Asistente de la Universidad del Valle. Miembro de la Unidad de Investigación en Salud Reproductiva de la Escuela de Enfermería Dra. Gladys Román de Cisneros, Universidad de Carabobo. Santiago de Cali, Colombia.

Correo electrónico: molano.maria@correounivalle.edu.co

ORCID: https://orcid.org/0000-0003-3931-3779 
importancia del Bienestar Espiritual para la humanización de los servicios de salud en el ámbito hospitalario, dado que contribuye a la mejora continua y a la garantía de calidad, preocupaciones relevantes para la bioética en el estudio del clima ético en las instituciones de salud.

Palabras clave: armonía; bienestar espiritual; disonancia; enfermería

Fecha de Recepción: 10 de septiembre de 2018 Fecha de Evaluación: 28 de octubre de 2018

Fecha de aceptación: 26 de novimebre de 2018 Publicación en Línea: 19 de diciembre de 2018

Cómo citar: Henao, A. M., Gomez Ramirez, O. Y., Molano-Pirazán, M. L., Fuentes Ramirez, A., \& Diaz-Heredia , L. P. (2019). Bienestar Espiritual en el ámbito Hospitalario: aportes para la humanización en salud. Revista Latinoamericana de Bioética, 19(36-1). https://doi.org/10.18359/ rlbi.3627

\section{Spiritual well-being in the hospital environment: contributions for health humanization}

Abstract: The objective of this article is to determine the spiritual well-being (SW) of nursing staff and their ethical contribution to health humanization. The method followed corresponds to the quantitative, descriptive and transversal approach. The sampling is not probabilistic, consisting of 148 nurses from two health institutions in the city of Bogotá (A and B). The SHALOM-3 instrument was applied to measure spiritual well-being (dissonance and harmony) in the personal, transcendental, environmental and communal domains. Regarding the results, the evaluation of the BE showed a high percentage of harmony in the two institutions; however, percentages of dissonance were found in institution B, in the transcendental (16\% vs. 22\%), environmental (12\% vs. $19 \%$ ) and personal (11\% vs. $18 \%$ ) domains. The importance of Spiritual Wellbeing for the humanization of health services in the hospital field is highlighted, since it contributes to a continuous improvement and quality assurance, both relevant concerns for bioethics in the study of the ethical climate in healthcare institutions.

Keywords: harmony; spiritual well-being; dissonance; nursing

\section{Bem-estar espiritual no âmbito hospitalar: contribuições para a humanização em saúde}

Resumo: $\mathrm{O}$ objetivo do presente artigo é determinar o bem-estar espiritual (BEE) da equipe de enfermagem e sua contribuição ética para a humanização em saúde. O método corresponde ao enfoque quantitativo, descritivo e transversal. A amostragem é não probabilística, constituída por 148 enfermeiros de duas instituições de saúde da cidade de Bogotá (A e B). Usou-se instrumento SHALOM-3 para medir o bem-estar espiritual (dissonância e harmonia) nos domínios pessoal, transcendental, ambiental e comunitário. Quanto aos resultados, a avaliação do BEE mostrou uma alta porcentagem de harmonia nas duas instituições; no entanto, encontraram-se porcentagens de dissonância na instituição B, nos domínios transcendental (16\% v. 22\%), ambiental (12\% v. $19 \%$ ) e pessoal (11\% v. 18\%). Destaca-se a importância do Bem-estar Espiritual para a humanização dos serviços de saúde no âmbito hospitalar, dado que contribui para a melhora contínua e a garantia de qualidade, preocupações relevantes para a bioética no estudo do clima ético nas instituições de saúde

Palabras-chave: harmonia; bem-estar espiritual; dissonância; enfermagem 


\section{Introducción}

El bienestar espiritual (BE), en el ámbito hospitalario, se demuestra en el trabajo de humanización de los servicios de salud que despliegan los diferentes actores del sistema como parte de sus procesos de mejoramiento continuo de la calidad (Zúniga Romero, 2016). Se reconoce que la humanización de la atención en salud inicia con la interacción de las personas en procesos de salud, enfermedad y muerte. Estas personas, a su vez, experimentan armonía y empatía como resultante del trabajo de bienestar espiritual en todas sus dimensiones, lo que se plantea como un aporte a la humanización para los servicios de salud y la reflexión bioética (Correa Zambrano, 2016).

Del mismo modo, el BE se describe como una naturaleza interconectiva que incluye la armonía del ser humano, originada en la congruencia entre el significado expresado y el vivido, los propósitos y los valores en la vida a nivel personal. En este sentido, para el presente estudio se acoge la propuesta teórica de Fisher (2010), que incluye los conceptos de armonía espiritual y disonancia espiritual. Al respecto, se define armonía espiritual como el estado de bienestar espiritual que se encuentra relacionado con la percepción acerca de los valores y propósitos de vida. Entretanto, la disonancia espiritual es entendida como la diferencia marcada entre las experiencias ideales y aquellas vividas en los dominios del BE (Fisher, 2010).

Las dos anteriores dimensiones pueden ser medidas a través del instrumento Shalom-3 (Gomez \& Fisher, 2003), el cual representa la esencia misma del BE. El nombre de este instrumento proviene de la palabra hebrea shalom, que significa "entereza, integridad, salud, paz, bienestar, plenitud, descanso y armonía" (Fisher, 2010, p. 108). Frente a ello, cabe anotar que Fisher, en la concepción inicial de BE, se basa en la Coalición Nacional sobre el Envejecimiento (NICA) para definirla como "la satisfacción de la vida en una relación con Dios, consigo mismo, con la comunidad y el medio ambiente que incentiva y celebra la plenitud" (Fisher, Barnes \& Marks, 2009, p. 11). Sin embargo, en estudios posteriores que incluyeron educadores y especialistas, Fisher definió la salud espiritual como "la dimensión fundamental de la salud que contribuye al BE de la población en general, permeando e integrando todas las otras dimensiones de la salud: física, mental, emocional y social" (Fisher, 1998, p. 25).

En este sentido, el BE es "un estado dinámico del ser, caracterizado por la medida en que las personas viven en armonía o disonancia en los dominios personal, comunal, ambiental y trascendental" (Fisher, 2010, p. 2). De esta manera, el dominio personal se define como la percepción de bienestar de cada persona consigo misma, con el significado, con el propósito y con los valores en la vida. El dominio comunal, por su parte, es la forma en la que se muestra la calidad y la profundidad de las relaciones interpersonales frente a la moral, la cultura y la religión; relaciones manifestadas en el amor, el perdón, la confianza, la esperanza y la fe en la humanidad. El dominio ambiental, en cambio, va más allá del cuidado de la vida y del entorno, en un sentido de admiración y asombro por la naturaleza, lo cual incluye la noción de unidad física y biológica con el medio ambiente. El dominio trascendental, finalmente, es la relación del yo con algo o alguien más además del nivel humano; es decir, una fuerza cósmica superior, la realidad transcendente o Dios.

En el ámbito hospitalario, se reconoce que el personal de enfermería brinda bienestar a las personas durante el cuidado que realiza en el ejercicio de la práctica profesional y, en respuesta, recibe una recompensa espiritual que refuerza los valores y ofrece la oportunidad de proyectarse personal y profesionalmente. Al darse este intercambio, se generan relaciones interpersonales que aportan significativamente a la visualización de los procesos de humanización en escenarios de atención en salud. En este sentido, se justifica el valor de la dimensión transpersonal de beneficiar al otro y enriquecerse en el proceso de dar cuidado (Haber et al., 2013; Watson, 2007), por lo que la idea expuesta sobre el BE es el resultado del compromiso de la disciplina de enfermería con el cuidado de la persona desde una concepción humanista que se evidencia en servicios de salud humanizados (Fisher, 2008). Desde esta perspectiva, el ser humano se aprecia en el valor de sí mismo y en la ética de sus comportamientos e interrelaciones con los otros, con la familia, la sociedad y la comunidad. La persona que da cuidado 
de enfermería se valora en su comportamiento, ya sea en las relaciones con la persona cuidada - que a veces se denomina paciente o cliente, con quien se relaciona individualmente-o como miembro de una familia o de un grupo comunitario, siempre respetando su individualidad, su valor y dignidad, sus derechos, sus necesidades, sus prioridades, su cultura, su historia y su ejercicio autónomo.

Por todo lo anterior, que considera el BE como un principio fundamental para el ejercicio bioético de la práctica de enfermería en el ámbito hospitalario, surge el propósito del presente estudio, el cual tiene como objetivo determinar el BE (en cuanto a disonancia o armonía) del equipo de enfermería de dos hospitales en la ciudad de Bogotá (Colombia), a través del instrumento Shalom-3, considerando la propuesta teórica de John Fisher e incluyendo la reflexión acerca del aporte del BE a la meta de la humanización en salud. Gracias a esta investigación, se propone la novedad y el aporte que ofrece el BE como expresión de la relación individualizada, en la cual se respeta el valor, la dignidad, los derechos, las prioridades y las necesidades de la persona que recibe el cuidado, lo cual garantiza la humanización de los servicios de salud (Andino Acosta, 2015). En la actualidad, la humanización de los servicios es una iniciativa que está inmersa en las propuestas para el mejoramiento de la calidad en salud con el propósito de motivar el planteamiento de nuevas perspectivas acerca de cómo abordar la dignidad humana, la protección y la calidad de vida de las personas con alteraciones de salud (Ministerio de Salud y Protección Social, 2016).

\section{Métodos}

El estudio se desarrolló desde un enfoque cuantitativo, descriptivo observacional, a partir de una medición que permitiera describir de manera transversal (en un solo momento de la línea del tiempo) el BE en el personal de enfermería de dos instituciones prestadoras de salud de la ciudad de Bogotá (Colombia). Esta medición se realizó bajo la aprobación del protocolo por parte del Comité de Ética en Investigación de la Facultad de Enfermería de la Universidad Nacional de Colombia y contó tanto con el aval de las instituciones de salud involucradas, como con el permiso del personal de enfermería dispuesto para la ejecución de la propuesta.

Para dar cumplimiento a los principios éticos de la investigación, los participantes fueron entrevistados de manera directa por el equipo de investigadoras luego de cumplir el protocolo de investigación y el respectivo consentimiento informado. Así mismo, para garantizar el principio de beneficencia y reciprocidad, el equipo de enfermería participó de manera voluntaria en talleres motivacionales en humanización por medio de narrativas de situaciones de enfermería y de mejora del clima laboral, planeados con las líderes y coordinadoras de enfermería de las instituciones de salud participantes.

\section{Participantes}

La población estuvo compuesta por el equipo de enfermería de dos instituciones proveedoras de salud (IPS), constituida por enfermeras(os) profesionales y técnicas(os), que correspondían a 250 personas. El muestreo fue no probabilístico, por lo que se contó con la participación de 148 personas que integraban el equipo de enfermería al momento del desarrollo del proyecto en las IPS participantes y que cumplieran fundamentalmente con dos criterios de inclusión: 1) ser trabajadores del área de enfermería con contrato de trabajo vigente y estable; y 2) que sus actividades laborales incluyeran cuidado directo de los pacientes. En cuanto a criterios de exclusión, fueron los funcionarios de enfermería que se encontraran realizando cargos administrativos de coordinación, supervisión o dirección de servicios, o que durante su labor no tuvieran contacto directo con el paciente.

Igualmente, se verificó que las instituciones en las cuales se llevaría a cabo el estudio hicieran parte del sistema de salud colombiano. Posteriormente los hospitales elegidos fueron caracterizados de la siguiente manera: la institución A es privada, declarada de carácter religioso y fundada en la inspiración de principios evangélicos. La institución B es un establecimiento público que brinda atención a los problemas de salud asociados a enfermedades pulmonares. Ambos hospitales son de tercer nivel de atención, por lo que cuentan con servicios de urgencias, internación y cirugía de alta complejidad. 


\section{Instrumentos}

El primer instrumento empleado fue un formato de datos sociodemográficos, en el cual se consideraron datos epidemiológicos de la muestra, tales como la edad, género, estado civil, institución de trabajo y servicio de actuación.

El segundo instrumento empleado fue una versión abreviada del instrumento Shalom-3, que evalúa el bienestar espiritual (Fisher, 2016). Dicha versión incluye tres componentes: A) evaluación de la salud espiritual, B) evaluación de la orientación de vida y C) evaluación de la percepción del enfermero sobre la ayuda dada a los pacientes. Tales componentes son evaluados a través de 20 ítems correspondientes y agrupados en los cuatro dominios (personal, comunal, ambiental y transcendental), por medio de tres preguntas a ser respondidas con escala tipo Likert de 1 (muy poco) a 5 (muchísimo).

Dentro del componente A del instrumento, se pueden evidenciar los ideales de salud espiritual para las personas por medio de la pregunta: “QQué tan importante es cada uno de los siguientes elementos para conseguir un estado ideal de salud espiritual?". Por su parte, en el componente B, se pregunta: “¿Cómo siente usted que los siguientes temas reflejen su experiencia personal la mayor parte del tiempo?". Finalmente, para enfermería, en el componente $\mathrm{C}$ se indaga en la pregunta: “QQué tanta ayuda cree que sus pacientes reciben de usted para desarrollar este aspecto de su vida?".

Frente a la versión abreviada Shalom-3, esta cuenta con propiedades psicométricas de validez y confiabilidad en el idioma inglés, resultado del trabajo sistemático y riguroso aplicado a cuatro estudios que soportan el modelo de Fisher (Gómez \& Fisher, 2003). Como resultado, el instrumento tiene un Alpha de Cronbach de 0.81 en la dimensión personal, de 0.82 en la dimensión comunal, de 0.86 en la dimensión ambiental y de 0.95 en la dimensión trascendental.

Previamente a la aplicación de dicho instrumento, se realizó su traducción al español, debido a que no se encontraba disponible en este idioma, en otras palabras, se realizó el proceso de adaptación transcultural, teniendo en cuenta la diversidad cultural, religiosa, étnica e idiomática del contexto colombiano. De este modo, el primer paso fue hacer el instrumento comprensible en cuanto a idioma y semántica, por lo que se hizo traducción al español y retraducción al idioma inglés, para posteriormente someterlo a pruebas de validez facial, de contenido y de constructo en una muestra de 143 enfermeros y 7 especialistas colombianos, con experiencia de cuidado directo al paciente, de ejercicio profesional y de docencia universitaria. Las pruebas de validez mostraron niveles de confiabilidad con Alfa de Cronbach de 0.95 e índice de Lawshe modificado CVI de 0.76 en la versión en español para la validez de contenido (Henao, Molano, Fuentes, Gómez \& Díaz Heredia, 2016).

En cuanto validez de apariencia, se hicieron cambios de redacción de algunos ítems sin perder el sentido original de los mismos, posterior a lo cual se obtuvo un índice de $\mathrm{V}$ de Aiken válido de $0.70-0.86$. Por su parte, los resultados de la validez facial presentaron valores superiores a 0.70 para un intervalo de confianza de $95 \%$ en 17 de los 20 ítems. De esta manera, se presentaron valores inferiores en tres ítems: los ítems 10 y 12 podían ser unificados y el ítem 20 presentó dificultades debido a la palabra "magia". Dado que esta palabra no fue comprensible en el contexto del español colombiano, se solicitó la autorización a Fisher para realizar el ajuste necesario y reemplazar el término por "maravilla", ya que se consideró que expresaba mejor la concepción de la naturaleza.

Con respecto a la validez de contenido de Shalom-3, esta fue establecida a través del índice de Lawshe modificado (CVI), el cual permite contar con un número reducido de expertos. Este modelo, modificado, es de fácil interpretación y está sustentado en la normalización y definición de un nuevo indicador del acuerdo entre los jueces (Tristán-López, 2008). Así, a través del CVI, se obtuvo un puntaje total del instrumento de 0.76 , ubicado en el intervalo 1 de $0.58-0.76$.

Con respecto a la validez de constructo (Soriano Rodríguez, 2014), se encontró a través de análisis factorial exploratorio que el instrumento cuenta con cuatro factores y que la varianza explicada es de 52.2. En suma, el instrumento presentó validez facial, de contenido y de constructo en el idioma 
español, lo que demuestra su utilidad en el contexto hispanohablante colombiano para determinar el $\mathrm{BE}$ de las personas.

Finalmente, el tercer instrumento empleado fue una escala tipo Likert de 5 puntos, a través de la cual se les preguntó a los participantes el nivel de contribución de diferentes estrategias que fomentan el BE en la persona, como por ejemplo la familia, la oración (rezar), caminatas, tiempo para relajamiento, ayudar a los otros, etc.

\section{Procedimiento}

La recolección de datos fue realizada para cada una de las instituciones en un período de trabajo de campo de seis meses. Dicha recolección se ejecutó a través de entrevista directa y aplicación de los instrumentos al personal de enfermería de los diferentes servicios de cada institución de salud. Se contó con la firma del consentimiento informado libre por parte de los participantes y las autorizaciones de las instituciones pertinentes. Así mismo, se garantizó la confidencialidad al mantener en un lugar seguro la información recolectada y al velar por los principios de propiedad intelectual.

\section{Análisis estadístico}

Las variables estudiadas fueron sometidas a un análisis exploratorio para identificar su distribución. En aquellas cuantitativas, se calcularon medidas de tendencia central (media) y de variabilidad (desviación estándar). Por su parte, para aquellas cualitativas, se calcularon proporciones. Para la normalización del $\mathrm{BE}$, se consideraron las respuestas de los 5 ítems evaluados en cada uno de los 4 dominios (personal, comunal, ambiental y trascendental), dando un total de 20 ítems. Posteriormente, las respuestas fueron clasificadas según los componentes del instrumento $(\mathrm{A}, \mathrm{B}, \mathrm{C})$ para finalmente calcular los porcentajes y promedios de respuesta por cada institución.

Para evaluar el BE como un todo, se calculó el porcentaje acumulado de respuestas para cada punto de la escala Likert en cada ítem de los dominios y para cada componente del instrumento, para luego recategorizar este porcentaje en "muy bajo", "bajo", "moderado", "alto" y "muy alto". Los valores medios de las respuestas de las enfermeras(os) en los
3 componentes, para cada uno de los 4 dominios del BE, se calcularon dividiendo la sumatoria de la puntuación de los ítems de cada dominio entre el número de ítems del dominio correspondiente.

Posteriormente, en relación con la disonancia espiritual, según lo propuesto por Fisher (2003), se explicó cuantitativamente como la diferencia en promedio mayor que 1,0 punto (más de 1 unidad de desviación estándar) entre los ideales (A) y la experiencia vivida (B) en cualquier dominio del BE, medido a través del instrumento Shalom-3. Las comparaciones por género y grupos de edad se realizaron usando la prueba de Chi-cuadrada o la prueba de Fisher, según fuera el caso. En las comparaciones, la significancia estadística fue considerada cuando $\mathrm{p}<0.05$.

Finalmente, fueron descritas las estrategias que fomentaban el BE en el personal de enfermería, discriminándolas por institución. Los datos fueron almacenados en una base de datos de Excel 2007 y posteriormente analizados estadísticamente en el programa Stata, versión $11.2^{\circ}$

\section{Resultados}

\section{Información sociodemográfica}

La muestra final estuvo compuesta por 148 participantes provenientes de dos hospitales, en su mayoría mujeres solteras en la franja etaria de 30 a 41 años. Ambos hospitales contaron con muestras similares y sin diferencias estadísticamente significativas con respecto a las características de estado civil y edad, por lo que solamente se aprecia diferencia entre hombres y mujeres, como se observa en la tabla 1.

\section{Instrumento Shalom-3}

En general, los datos muestran porcentajes de armonía altos, como se evidencia en la tabla 2.

De acuerdo con las tablas, se muestran los valores promedio de las respuestas obtenidas para el hospital A y el hospital B sobre 5 puntos. Así, los dominios personal, comunal y trascendental tienen valores mayores a 4, lo cual los ubica en un valor de "mucho", es decir, un nivel de armonía que tienen los profesionales de enfermería entre los ideales, la 
Tabla 1. Información sociodemográfica del personal de enfermería según institución. Bogotá, Colombia, 2015 $(n=148)$

\begin{tabular}{|c|c|c|c|}
\hline Característica & $\begin{array}{c}\text { Hospital A } \\
(n=70)\end{array}$ & $\begin{array}{c}\text { Hospital B } \\
(n=78)\end{array}$ & P valor \\
\hline \multicolumn{4}{|l|}{ Género, n (\%) } \\
\hline Femenino & $68(97.1)$ & $65(83.3)$ & \multirow{2}{*}{$0.006^{*}$} \\
\hline Masculino & $2(2.9)$ & $13(16.7)$ & \\
\hline \multicolumn{4}{|l|}{ Estado civil, n (\%) } \\
\hline Soltero & $29(41.4)$ & $32(41)$ & \multirow{6}{*}{$0.587^{\star}$} \\
\hline Casado & $23(32.9)$ & $26(33.3)$ & \\
\hline Divorciado & 0 & $3(3.8)$ & \\
\hline Viudo & $1(1.4)$ & $1(1.3)$ & \\
\hline Unión libre & $11(15.7)$ & $13(16.7)$ & \\
\hline Separado & $6(8.6)$ & $3(3.8)$ & \\
\hline \multicolumn{4}{|l|}{ Franja etaria, n (\%) } \\
\hline 18 a 29 & $21(30)$ & $27(35)$ & \multirow{3}{*}{$0.815^{* \star}$} \\
\hline 30 a 41 & $28(40)$ & $28(36)$ & \\
\hline 42 a 53 & $21(30)$ & $23(29)$ & \\
\hline
\end{tabular}

Fuente: datos de la investigación. *Test exacto de Fisher. **Prueba de hipótesis Chi cuadrado.

Tabla 2. Valores medios de las respuestas del personal de enfermería según los dominios del bienestar espiritual y por institución. Bogotá, Colombia, 2015 ( $n=148)$

\begin{tabular}{l|ccc|ccc|ccc|ccc}
\multicolumn{1}{c|}{ Dominios } & \multicolumn{3}{c|}{ Personal } & \multicolumn{3}{c|}{ Comunal } & \multicolumn{3}{c|}{ Ambiental } & \multicolumn{3}{c}{ Trascendental } \\
Institución & A & B & C & A & B & C & A & B & C & A & B & C \\
\hline Hospital A & 4.6 & 4.5 & 4.2 & 4.3 & 4.2 & 4.1 & 4.2 & 4.1 & 3.8 & 4.5 & 4.4 & 4.0 \\
\hline Hospital B & 4.6 & 4.5 & 4.2 & 4.4 & 4.3 & 4.1 & 4.2 & 4.2 & 3.8 & 4.4 & 4.3 & 4.1 \\
\hline
\end{tabular}

Fuente: datos de la investigación. Componentes instrumento: A (ideal), B (experiencia vivida), C (ayuda)

Categorías de evaluación escala Likert: 1. Muy Poco 2. Poco 3. Moderado 4. Mucho 5. Muchísimo.

experiencia vivida y la ayuda. El único valor que muestra un valor inferior a 4 es el dominio ambiental, en el ítem de "ayuda a otros".

En los resultados, se resalta una proporción baja de disonancia, que invita a reflexionar en la percepción de armonía en los diferentes dominios, siendo el más armonioso el dominio comunal. En este sentido, se presenta una mayor proporción de disonancia en el hospital B. A continuación, se describen los resultados obtenidos para cada dominio del instrumento, discriminando los datos para el hospital A y el hospital B. En la figura 1, se encuentran esquematizados los porcentajes de la variable disonancia espiritual para cada dominio según la institución.

Dominio personal: Los resultados en el hospital A mostraron un porcentaje de disonancia de $13 \%$ $(\mathrm{n}=8)$, todos los casos fueron del género femenino, el $50 \%$ tenían una edad entre 30 y 41 años, y no hubo diferencias significativas entre los grupos $(\mathrm{p}=0.86)$ al comparar las otras franjas etarias para 
este resultado. Por su parte, en el hospital B, hubo un porcentaje de disonancia de $18 \%(\mathrm{n}=11)$, la mayoría $(n=8)$ fueron del género femenino $(73 \%)$ con edades entre 30 y 41 años (45\%), y no hubo diferencias significativas $(\mathrm{p}=0.41)$.

Dominio comunal: En el hospital A, la disonancia fue de $6,4 \%(n=4)$, todos los casos en mujeres, $50 \%$ con edades entre 30 y 41 años, y no hubo diferencias significativas entre los grupos $(\mathrm{p}=0.88)$. Por su parte, en el hospital B, en el dominio comunal la disonancia fue de $6,4 \%(n=4)$, la mitad de los casos (50\%) fueron mujeres, $75 \%$ con edades entre 30 y 41 años, $y$ no hubo diferencias significativas $(\mathrm{p}=0.23)$.

Dominio ambiental: En el hospital A hubo 12\% de casos de disonancia $(n=7)$, entre los cuales 6 fueron del género femenino (86\%), la mayoría tenía edades entre 30 y 41 años (43\%), y no hubo diferencias significativas entre grupos $(\mathrm{p}=0.99)$. Para el hospital B, el 19\% presentaron disonancia $(n=12)$, el $67 \%$ fueron del género femenino, el $42 \%$ con edades entre 30 a 41 años, y sin diferencias estadísticamente significativas $(\mathrm{p}=0.97)$.

Dominio trascendental: En el hospital A, la disonancia fue de $16 \%(\mathrm{n}=10)$, el $90 \%$ en mujeres, el $50 \%$ estaban entre 30 y 41 años, seguido del 30\% entre 18 a 29 años; no hubo diferencias significativas $(\mathrm{p}=0.7)$. Para el hospital B, la disonancia fue del $22 \%$ $(\mathrm{n}=15)$, mayormente del género femenino $(73 \%)$, con una edad entre 30 y 41 años (47\%); no hubo diferencias significativas $(\mathrm{p}=0.44)$.

Los valores del Alfa de Cronbach se obtuvieron con el objetivo de establecer la confiabilidad de los datos para cada dominio, especificando los componentes A, B y C del instrumento. La figura 1 muestra el porcentaje de disonancia según institución por dominios personal, comunal, ambiental y trascendental.

En cuanto a las estrategias que los participantes seleccionaron como las usadas para promover el bienestar espiritual, se destacan en mayor proporción para el hospital A estar feliz y ayudar a otros con un $60 \%$, seguida de realizar actividades en familia con un $56,7 \%$. Entretanto, para el hospital B, se destacan estar feliz con un 54\%, ayudar a otros con un $47,2 \%$ y realizar actividades en familia con un $58,1 \%$. Así mismo, los participantes de los dos hospitales otorgaron una importancia a la espiritualidad en su vida mayor al 50\%. Por otro lado, las estrategias calificadas como poco usadas por los participantes son practicar meditación y tener tiempo para relajarse.

\section{Discusión}

Los resultados del presente estudio reafirman la propuesta teórica de Fisher (Gomez \& Fisher, 2003)

Figura 1. Porcentaje de disonancia según institución por dominios personal, comunal, ambiental y trascendental. Bogotá-Colombia

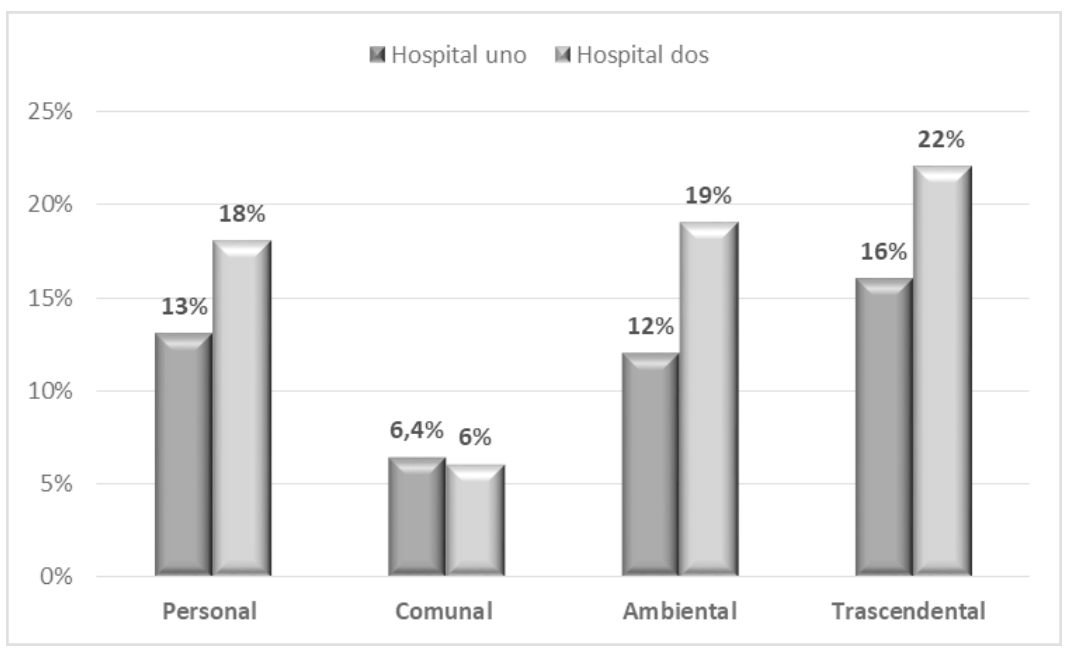

Fuente: datos de la investigación. 
en la cual el BE se ubica como un constructo que integra los aspectos personales, de interacción con otros y de trascendencia en la búsqueda de la armonía y el propósito de vida en los individuos. Estos atributos conceptuales se consideran de interés en el abordaje de la humanización de los servicios de salud que vincula la dignidad humana como propósito central de la atención en salud (Ministerio de Salud y Protección Social, 2016).

De acuerdo con los componentes del BE en la propuesta de Fisher (Fisher, 2010), los resultados dan cuenta de que el dominio trascendental presentó mayor disonancia ( $16 \%$ y $22 \%$, respectivamente para los hospitales A y B), es decir, que los profesionales de enfermería no refieren armonía en la relación de sí mismos con algo o alguien de naturaleza cósmica superior, una realidad trascendente o Dios. Este dominio está relacionado con la fe religiosa, pero no se circunscribe únicamente a esta. La fe religiosa se manifiesta como una fuente de energía que las fortalece e impulsa para el trabajo diario. Inclusive, la espiritualidad puede ser benéfica para el desempeño del profesional en el ambiente de trabajo, visto que esta puede influir de forma positiva en aspectos como el compromiso organizacional, la superación de las adversidades en el trabajo, la realización personal de los profesionales, etc. (Abbasi, Farahani-Nia, Mehrdad \& Hamid Haghani, 2014).

Lo anterior motiva a continuar estudiando aspectos que puedan ampliar la comprensión del concepto de espiritualidad para el personal de enfermería (Ramezani, Ahmadi, Mohammadi, \& Kazemnejad, 2014), según el cual los atributos que mejoran la comprensión del concepto trascendental son la presencia sanadora, el uso terapéutico de sí mismo y el sentido intuitivo. Al respecto, en un estudio cualitativo con 31 participantes (Ødbehr, Kvigne, Hauge \& Danbolt, 2014), emergieron tres temas importantes en la consideración de las necesidades espirituales para enfermería que pueden hacer parte del dominio trascendental: la necesidad de paz y serenidad, la necesidad de amor y proximidad, y la necesidad de expresar creencias (Hussey \& Phil, 2009). Todos estos atributos pueden ser estudiados a profundidad con nuevas investigaciones de abordaje cualitativo dentro del concepto del BE asociado al cuidado de enfermería. En este sentido, es importante resaltar la importancia de la relación ética, en la que los valores de cada persona (paciente y enfermero) deben ser conocidos y considerados, lo que exige del personal de enfermería un conocimiento de los valores y principios que sustenten el ejercicio profesional de la enfermería como práctica social (Garzón Alarcón, 2005).

Por su parte, el dominio comunal presentó resultados bajos de disonancia en el personal de enfermería de las dos instituciones. Un bajo nivel de disonancia en el dominio comunal indica que las personas son capaces de generar relaciones interpersonales significativas que favorezcan el trabajo en equipo y el clima laboral, que se podría relacionar con las características positivas del trabajo de enfermería en la práctica clínica. La relación interpersonal que se establece utiliza efectivamente atributos de la interacción como la confianza y la empatía, los cuales son desarrollados por la enfermera experta en un ambiente de respeto por la dignidad del paciente como persona, lo que demuestra su capacidad de sentir con este, de ponerse en su lugar, y de establecer tiempos y espacios para que se sienta comprendido y pueda lograr por sí mismo su bienestar (González Ortega, 2007). Estas relaciones interpersonales fortalecen el vínculo entre el paciente y la enfermera como elementos fundamentales para brindar un cuidado de aporte a la humanización a los servicios de salud, por lo que se hace evidente entonces el vínculo entre la bioética, los valores espirituales y el concepto de cuidado (Rudolfsson, Berggren \& Da Silva, 2014).

En cuanto al dominio personal, los porcentajes de disonancia que arrojaron los resultados fueron bajos, pero llama la atención que, al igual que en los otros dominios, se dio en mayor medida dentro del hospital B, con especial relevancia dentro del género femenino (para ambos hospitales). A partir de este resultado, se reflexiona que cada individuo experimenta cambios que pueden tener repercusiones en su vida personal, en su proyecto de vida y en cómo están asentados sus valores (Friedemann, Mouch \& Racey, 2002), fenómeno que durante el ciclo vital recibe un mayor énfasis en la tercera y cuarta década (Dezorzi \& Crossetti, 2008). 
De igual forma, se requieren estudios que aborden los factores relacionados con este dominio, que podrían relacionarse con el ambiente laboral (Chung, Wong \& Chan, 2007) y contrastar con estos la calidad de las actividades de cuidado (Carrillo García et al., 2014). Estos factores motivan preocupaciones relevantes para la bioética en el estudio del clima ético en las instituciones, lo cual incluye la calidad (acceso y seguridad), los cambios en los sistemas de salud, las presiones organizacionales $y$, por último, la posibilidad de que los profesionales realicen una reflexión para dar respuesta a la complejidad de los problemas reales en el ámbito de las organizaciones de salud (Arango Bayer, 2011).

Finalmente, el dominio ambiental fue el segundo más disonante dentro de la muestra. Este resultado invita a continuar estudiando en el personal de enfermería la promoción de la armonía con el ambiente, la satisfacción laboral y su repercusión en la calidad de los servicios de salud. La disonancia puede asociarse con condiciones laborales que se evidencian en instituciones de salud, donde aspectos como el tipo de contratación y el clima laboral pueden ser variables que afecten el BE (Juárez-Aduata, 2012).

Ahora bien, en cuanto a las estrategias que usa el personal de enfermería para promover el BE, estas se enfocan principalmente en mejorar la espiritualidad. Incluso, las prácticas que usan las profesionales de enfermería son congruentes con hallazgos anteriores que muestran que los participantes ayudan a los demás, escuchan música, oran a solas, le buscan sentido a la enfermedad y el sufrimiento o buscan estar en relación con los otros y con el ser superior. Lo anterior señala la presencia evidente de valores que fortalecen el ejercicio ético y bioético del cuidado, entre los que se mencionan creencias espirituales, fe y esperanza, asociadas a conceptos como sentido de vida, relaciones, transcendencia y prácticas religiosas (Garzón Alarcón, 2005). Esta información también se reafirma con un estudio que sugiere que la espiritualidad puede ser beneficiosa para la calidad de vida y el bienestar físico y mental (Castelo-Branco, Brito \& Fernandes-Sousa, 2014; Nascimento et al., 2016).

Es así que las estrategias que usa el personal de enfermería tienen un efecto sobre las actitudes de las enfermeras hacia la atención espiritual, compromiso profesional y la ética del cuidado, la cual reconoce la importancia de la cercanía a la realidad percibida, la presencia del otro, de sus facciones, sus expresiones, su actitud, su necesidad y su vulnerabilidad, demostrando así su capacidad de ayuda frente a la fragilidad (Franco Peláez, 2009). Esta premisa describe las actitudes de las enfermeras hacia la atención espiritual, lo que permite mejorar su compromiso profesional y la capacidad de prestar cuidados congruentes con los propósitos de la humanización de los servicios de salud (Chiang, Lee, Chu, Han \& Hsiao, 2016; Koening, 2009), lo cual se refleja en el nivel de $\mathrm{BE}$.

De todos modos, y pese a lo anterior, los resultados muestran que los profesionales de enfermería utilizan en menor medida las prácticas religiosas como estrategias para fomentar el $\mathrm{BE}$, tales como visitar al sacerdote o al líder religioso, los cuales pueden ser importantes recursos para hacer frente al proceso de enfermedad que experimenta la persona a su cuidado e incluso, contribuir a la disminución de la aparición o abordaje de una patología mental que se presente en algunos casos (Koening, 2009).

\section{Conclusiones}

Estudiar las dimensiones del BE con la propuesta teórica de Fisher permitió cumplir los objetivos del presente estudio y de esta manera proponer que el constructo de BE es un aporte a la generación del valor social que les asiste a los agentes para el mejoramiento continuo de la calidad en salud y la humanización de los servicios ofrecidos. Por medio de esta investigación, se motiva a que estudios posteriores utilicen el instrumento Shalom-3 para dar a conocer las percepciones del talento humano en salud, para que los diversos actores del sistema reconozcan que para lograr la humanización de los servicios de salud se requiere entender las consideraciones de las personas acerca de su bienestar personal, comunal, ambiental y trascendental, de tal forma que sus comportamientos demuestren el valor social de su labor como agentes de cuidado. Si las instituciones y los agentes incorporaran los cuatro dominios del bienestar espiritual (personal, comunal, ambiental y trascendental), harían evidente uno de los talentos que hacen parte del marco de los principios éticos y bioéticos para la humanización de los servicios de 
salud, ya que se haría evidente el enfoque humano y centrado en las personas, señalados en los modelos de excelencia en gestión (Government of Western Australia, 2013).

Así, es necesario continuar realizando investigaciones sobre este fenómeno en la práctica asistencial de enfermería, de modo que puedan ayudar en el entendimiento de las disonancias considerando el BE como un constructo de gran contribución tanto para el área asistencial y personal de los enfermeros y enfermeras (aportando a la conducción del cuidado humano dentro de su rol profesional, que envuelve un sentido bioético profundo), como en la gestión de recursos humanos de enfermería, que en últimas se refleja en un aporte concreto a la humanización de los servicios de salud.

\section{Referencias}

Abbasi, M., Farahani-Nia, M., Mehrdad, N. \& Hamid Haghani, A. (2014). Nursing students' spiritual well-being, spirituality and spiritual care. Iranian Journal of Nursing and Midwifery Research, 19(3), 242-247. Retrieved from https://www.ncbi.nlm.nih.gov/pubmed/24949061

Andino Acosta, C. A. (2015). Bioética y humanización de los servicios asistenciales en la salud. Revista Colombiana de Bioética, 10(1), 38-64. Recuperado de http:// www.bioeticaunbosque.edu.co/publicaciones/Revista/ Rev101/art3_carlosandino.pdf

Arango Bayer, G. L. (2011). Clima ético en organizaciones de salud: revisión de la literatura. Revista Colombiana de Bioética, 6(1), 145-164. Recuperado de http://www. bioeticaunbosque.edu.co/publicaciones/Revista/Revista12/art9.pdf

Carrillo García, C., Ríos Rísquez, M. I., Castaño Molina, M. Á., Nicolás Vigueras, M. D., Arnau Sánchez, J. y Martínez Roche, M. E. (2014). Estudio exploratorio de satisfacción laboral de los profesionales de enfermería generalistas y especialistas (matrona). Cultura de los Cuidados, 18(39), 105-114. https://doi.org/10.7184/ cuid.2014.39.12

Castelo-Branco, M. Z., Brito, D. e Fernandes-Sousa, C. (2014). Necessidades espirituais da pessoa doente hospitalizada: revisão integrativa. Aquichan, 14(1), 100-08. https://doi.org/10.5294/aqui.2014.14.1.8

Chiang, Y. C., Lee, H. C., Chu, T. L., Han, C. Y. \& Hsiao, Y. C. (2016). The impact of nurses' spiritual health on their attitudes toward spiritual care, professional commitment, and caring. Nursing Outlook, 64(3), 215-224. https://doi.org/10.1016/j.outlook.2015.11.012
Chung, L. Y. F., Wong, F. K. Y. \& Chan, M. F. (2007). Relationship of nurses' spirituality to their understanding and practice of spiritual care. Journal of Advanced Nursing, 58(2), 158-170. https://doi.org/10.1111/j.13652648.2007.04225.x

Correa Zambrano, M. L. (2016). La humanización de la atención en los servicios de salud: un asunto de cuidado. Revista Cuidarte, 7(1), 1227-1231. https:/doi. org/10.15649/cuidarte.v7i1.300

Dezorzi, L. W. y Crossetti, M. de G. O. (2008). La espiritualidad en el cuidado de sí para profesionales de enfermería en terapia intensiva. Revista Latino-Americana de Enfermagem, 16(2). https://doi.org/10.1590/S010411692008000200007

Fisher, J. W. (1998). Spiritual health: its nature and place in the school curriculum [Tesis de Ph.D.]. Universidad de Melbourne. Retrieved from https://minerva-access. unimelb.edu.au/handle/11343/39206

Fisher, J. W. (2008). Impacting teachers' and students' spiritual well-being. Journal of Beliefs \& Values, 29(3), 253-261. https://doi.org/10.1080/13617670802465789

Fisher, J. W. (2010). Development and Application of a Spiritual Well-Being Questionnaire Called Shalom. Religions, 1(1), 105-121. https://doi.org/10.3390/rel1010105

Fisher, J. W. (2016). Selecting the Best Version of Shalom to Assess Spiritual Well-Being. Religions, 7(5). https://doi. org/10.3390/rel7050045

Fisher, J. W., Barnes, L. P. \& Marks, G. (2009). Pre-service teachers' spiritual well-being across time and faiths: Implications for religious education. Religious Education Journal of Australia, 25(2), 10-16. Retrieved from https:// www.researchgate.net/publication/256498694_Fisher_ JW_Barnes_LP_Marks_G_2009_Pre-service_teachers'spiritual_well-being_across_time_and_faiths_ Implications_for_religious_education_Religious_Education_Journal_of_Australia_25210-16

Franco Peláez, Z. R. (2009). El conocimiento de la bioética como ética del cuidado: un imperativo para la formación en Trabajo Social. Revista Eleuthera, 3, 106-124. Recuperado de http://vip.ucaldas.edu.co/eleuthera/ downloads/Eleuthera3_4.pdf

Friedemann, M. L., Mouch, J. \& Racey, T. (2002). Nursing the spirit: The Framework of Systemic Organization. Journal of Advanced Nursing, 39(4), 325-332. https:// doi.org/10.1046/j.1365-2648.2002.02293.x

Garzón Alarcón, N. (2005). Ética profesional y teorías de enfermería. Aquichan, 5(1), 64-71. Recuperado de http:// aquichan.unisabana.edu.co/index.php/aquichan/article/view/60/124

Gomez, R. \& Fisher, J. W. (2003). Domains of spiritual well-being and development and validation of the 
Spiritual Well-Being Questionnaire. Personality and Individual Differences, 35(8), 1975-1991. https://doi. org/10.1016/S0191-8869(03)00045-X

González Ortega, Y. (2007). La enfermera experta y las relaciones interpersonales. Aquichan, 7(2), 130-138. Recuperado de http://www.redalyc.org/articulo. oa?id=74107205

Government of Western Australia. (2013). Western Australian Strategic Plan for Safety and Quality in Health Care 2013-2017. Retrieved from https://ww2. health.wa.gov.au/ /media/Files/Corporate/Reports and publications/PDF/WASafetyandQualityStrategicPlan2013-2017.pdf

Haber, J. R., Grant, J. D., Sartor, C. E., Koenig, L. B., Heath, A. \& Jacob, T. (2013). Religion/spirituality, risk, and the development of alcohol dependence in female twins. Psychology of Addictive Behaviors, 27(3), 562572. https://doi.org/10.1037/a0031915

Henao, A., Molano, M. L., Fuentes, A., Gómez, O. \& Díaz Heredia, L. P. (2016). Shalom una herramienta valida para medir el cuidado espiritual. En L. P. Díaz Heredia (Ed.), Experiencias de formación, investigación y práctica en el cuidado de enfermería (pp. 211 -223) (1a ed). Universidad Nacional de Colombia.

Hussey, T. \& Phil, M. A. D. (2009). Nursing and spirituality. Nursing Philosophy, (10), 71-80. https://doi.org/10.1111/ j.1466-769X.2008.00387.x

Juárez-Aduata, S. (2012). Clima organizacional y satisfacción laboral. Revista Médica del Instituto Mexicano del Seguro Social, 50(3), 307-314. Recuperado de https:// www.redalyc.org/pdf/4577/457745495014.pdf

Koening, H. (2009). Research on Religion, Spirituality, and Mental Health: A Review. The Canadian Journal of Psychiatry, 54(5), 283-292. https://doi.org/10.1177/ 070674370905400502

Ministerio de Salud y Protección Social. (2016). Plan Nacional de Mejoramiento de la Calidad en Salud (PNMCs):
Plan Estratégico 2016-2021. Ministerio de Salud y Protección Social. Recuperado de https://www.minsalud.gov.co/sites/rid/Lists/BibliotecaDigital/RIDE/DE/ CA/Plan-nacional-de-mejoramiento-calidad.pdf

Nascimento, L. C., de Oliveira, F. C. S., Santos, T. de F. M., Pan, R., Flória-Santos, M., Alvarenga, W. e. \& Rocha, S. M. M. (2016). Atenção às necessidades espirituais na prática clínica de enfermeiros. Aquichan, 16(2), 179192. https://doi.org/10.5294/aqui.2016.16.2.6

Ødbehr, L., Kvigne, K., Hauge, S. \& Danbolt, L. J. (2014). Nurses' and care workers' experiences of spiritual needs in residents with dementia in nursing homes: a qualitative study. BMC Nursing, 13(1). https://doi. org/10.1186/1472-6955-13-12

Ramezani, M., Ahmadi, F., Mohammadi, E. \& Kazemnejad, A. (2014). Spiritual care in nursing: A concept analysis. International Nursing Review, 61(2), 211-219. https://doi. org/10.1111/inr.12099

Rudolfsson, G., Berggren, I. \& da Silva, A. B. (2014). Experiences of Spirituality and Spiritual Values in the Context of Nursing - An Integrative Review. The Open Nursing Journal, 8, 64-70. https://doi. org/10.2174/1874434601408010064

Soriano Rodríguez, A. M. (2014). Diseño y validación de instrumentos de medición. Diá-logos, (14), 19-40. https://doi.org/10.5377/dialogos.v0i14.2202

Tristán-López, A. (2008). Modificación al modelo de Lawshe para el dictamen cuantitativo de la validez de contenido de un instrumento objetivo. Avances en medición, 6(1), 37-48. Recuperado de https://dialnet.unirioja.es/servlet/ articulo? codigo $=2981185$

Watson, J. (2007). Theory of Human Caring. En M. Smith \& M. Parker (Eds.), Nursing Theories and Nursing Practice (p. 347). Philadelphia: FA Davis Company.

Zúñiga Romero, M. (2016). Humanizar la Salud una propuesta desde el caribe colombiano: El caso Barranquilla. Barranquilla: Universidad del Norte. 